OPEN ACCESS

Edited by:

Yilong Wang,

Capital Medical University, China

Reviewed by:

Cristiano Capurso,

University of Foggia, Italy

Gentian Vyshka,

University of Medicine, Tirana, Albania

*Correspondence:

Xudong Li

lixd73cj@163.com

Specialty section:

This article was submitted to

Aging Psychiatry,

a section of the journal

Frontiers in Psychiatry

Received: 29 March 2021

Accepted: 09 June 2021

Published: 16 July 2021

Citation:

Li X, Shen M, Jin Y, Jia S, Zhou Z, Han Z, Zhang $X$, Tong $X$ and Jiao J (2021) The Effect of Cerebral Small Vessel Disease on the Subtypes of Mild Cognitive Impairment.

Front. Psychiatry 12:685965. doi: 10.3389/fpsyt.2021.685965

\section{The Effect of Cerebral Small Vessel Disease on the Subtypes of Mild Cognitive Impairment}

\author{
Xudong $\mathrm{Li}^{1,2 *}$, Miaoxin Shen ${ }^{3}$, Yi Jin ${ }^{4}$, Shuhong $\mathrm{Jia}^{4}$, Zhi Zhou ${ }^{4}$, Ziling Han ${ }^{1}$, \\ Xiangfei Zhang ${ }^{4}$, Xiaopeng Tong $^{3}$ and Jinsong Jiao ${ }^{4}$

\begin{abstract}
${ }^{1}$ Department of Cognitive Disorder, Beijing Tiantan Hospital, Capital Medical University, Beijing, China, ${ }^{2}$ China National Clinical Research Center for Neurological Diseases, Beijing, China, ${ }^{3}$ Medical School, Xizang Minzu University, Xianyang, China, ${ }^{4}$ Department of Neurology, China-Japan Friendship Hospital, Beijing, China
\end{abstract}

Objectives: Cerebral small vessel disease (CSVD) is the most common vascular cause of dementia, and mild cognitive impairment $(\mathrm{MCl})$ is an intermediate state between dementia and normal cognitive aging. The present study investigated the main imaging features of CSVD on different $\mathrm{MCl}$ subtypes in memory clinics.

Methods: A total of 236 patients with $\mathrm{MCl}$ and 85 healthy controls were included. One hundred nine amnestic $\mathrm{MCl}$-multiple domains (amMCl), 38 amnestic $\mathrm{MCl}$-single domain (asMCl), 36 non-amnestic $\mathrm{MCl}$-multiple domains (namMCl), and 53 non-amnestic $\mathrm{MCl}$ single domain (nasMCl) patients were diagnosed. All participants were evaluated with the cognitive assessments and imaging features including white matter hyperintensity (WMH), enlarged perivascular spaces (EPVS), cerebral microbleeds (CMBs), and cerebral atrophy according to a standard procedure.

Results: The patients with amMCl, namMCl, and nasMCl had more high-grade basal ganglia EPVS compared with healthy controls, while the percentages of high-grade basal ganglia EPVS in the patients with amMCl were also more than those in patients with asMCl, namMCl, and nasMCl. There were more high-grade centrum semiovale EPVS in patients with amMCl in comparison with all other groups. The patients with amMCl and namMCl had more percentages of severe deep and periventricular $\mathrm{WMH}$ and deep CMBs compared with healthy controls. All MCl groups had higher scores of the medial temporal lobe atrophy than healthy controls, whereas the scores of the amMCl group were also higher than those of the namMCl and nas $\mathrm{MCl}$ groups.

Conclusions: There were varied neuroimaging features of CSVD including cerebral atrophy in different $\mathrm{MCl}$ groups, which meant that vascular mechanism contributed to the prodromal stage of dementia.

\footnotetext{
Keywords: mild cognitive impairment, cerebral small vessel disease, MRI, white matter hyperintensity, enlarged perivascular spaces, cerebral microbleed, cerebral atrophy
}

\section{INTRODUCTION}

Dementia has become an important health problem among the aging population in China with 249.49 million people aged 60 years or older. Overall age-adjusted and sexadjusted prevalence was estimated to be $6.0 \%$ for dementia, 3.9\% for Alzheimer's disease $(\mathrm{AD})$, and $1.6 \%$ for vascular dementia in people aged 60 years or older in China (1). 
Mild cognitive impairment (MCI) is considered as an intermediate state between dementia and normal cognitive aging with prevalence of $15.5 \%$ and could provide important information about the population at risk for developing dementia $(1,2)$. The concept of MCI was expanded to four subtypes, namely, amnestic MCI-single domain (asMCI), amnestic MCImultiple domains (amMCI), non-amnestic MCI-single domain (nasMCI), and non-amnestic MCI-multiple domains (namMCI), which differ in etiology and outcome. Amnestic MCI (aMCI) is thought to have a high likelihood of progressing to AD, especially amMCI. Non-amnestic MCI (naMCI) is assumed to have a higher likelihood of progressing to a non-AD dementia $(2,3)$. Cerebral small vessel disease (CSVD) is a disorder of the brain's small perforating arterioles, capillaries, and probably venules that causes various lesions that are seen on pathological examination or brain imaging with magnetic resonance imaging (MRI) or computed tomography (CT). Typical CSVD includes white matter hyperintensities (WMH), enlarged perivascular spaces (EPVS), cerebral microbleeds (CMBs), and so on (4-6). Brain atrophy occurs with the usual aging process with heterogeneous pathological changes. Brain atrophy was thought to have a close relationship with neurodegenerative diseases, but many studies reported an association between atrophy and CSVD (4). Neurodegenerative diseases such as AD commonly coexist with cerebrovascular disease in older people, especially CSVD. CSVD could cause cognitive impairments and is a common cause of dementia, but the relationship has been questioned between CSVD and the various MCI types.

The purpose of the present study was to investigate the main imaging features of CSVD on different MCI subtypes.

\section{MATERIALS AND METHODS}

\section{Subjects}

Three hundred twenty-one subjects were recruited from the memory clinics between 2015 and 2019. All participants underwent routine assessments, including standardized history taking, physical and neurological examinations, necessary laboratory tests, and an MRI scan. Of these participants, 236 of them were diagnosed with MCI and fulfilled the following criteria: (1) cognitive complaint, preferably corroborated by an

\footnotetext{
Abbreviations: AD, Alzheimer's disease; ADL, activities of daily living; aMCI, amnestic mild cognitive impairment; amMCI, amnestic mild cognitive impairment-multiple domains; asMCI, amnestic mild cognitive impairmentsingle domain; BG, basal ganglia; BNT, Boston Naming Test; CDR, Clinical Dementia Rating Scale; CDT, Clock Drawing Test; CMBs, cerebral microbleeds; CSO, centrum semiovale; CSVD, cerebral small vessel disease; CT, Computed Tomography; DWMH, deep white matter hyperintensities; EPVS, enlarged perivascular spaces; FLAIR, fluid attenuated inversion recovery sequence; GCA-F, global cortical atrophy scale-frontal; MMSE, Mini Mental State Examination; MoCA, Montreal Cognitive Assessment; MRI, Magnetic Resonance Imaging; MTA, medial temporal lobe atrophy; naMCI, non-amnestic mild cognitive impairment; namMCI, non-amnestic mild cognitive impairmentmultiple domains; nasMCI, non-amnestic mild cognitive impairment-single domain; PA, posterior atrophy; PV WMH, periventricular white matter hyperintensities; RAVLT, Rey Auditory Verbal Learning Test; ROCF, ReyOsterrieth complex figure; SCWT, Stroop Color Word Test; SWI, susceptibilityweighted imaging; TMT-A, Trail Making Test A; TMT-B, Trail Making Test B; $\mathrm{WMH}$, white matter hyperintensities.
}

informant; (2) objective cognitive impairment, quantified as a performance score more than 1.5 standard deviation (SD) below the appropriate mean on one cognitive test of any domain; (3) essentially intact activities of daily living (ADL); and (4) no dementia $(2,3)$. Subjects with a score $<1.5 \mathrm{SD}$ from the mean on a learning measure, immediate or delayed recall, or recognition on the Rey Auditory Verbal Learning Test (RAVLT) (7) or immediate or delayed recall on the Rey-Osterrieth complex figure (ROCF) (7) were classified as having aMCI. Subjects with a score $<1.5 \mathrm{SD}$ from the mean on at least one test of attention, executive function, language facilities, and visuospatial capacity, but no memory impairment, were classified as having naMCI. Subjects with aMCI were subclassified as asMCI if they only had impairment in memory and as amMCI if they also had impairments in non-memory domains. Subjects with naMCI were subclassified as nasMCI if they only had impairment in one domain of non-memory domains and as namMCI if they also had impairments in two or above domains of non-memory domains. A total of 109 amMCI, 38 asMCI, 36 namMCI, and 53 nasMCI patients were included in the study.

Eighty-five subjects were considered as healthy controls. The inclusion criteria for the controls were as follows: (1) almost normal cognitive functions verified by informants; (2) the Mini Mental State Examination (MMSE) scores equal to or above 26 (8); (3) intact ADL; and (4) a Clinical Dementia Rating Scale (CDR) score of 0 (9). The exclusion criteria for the patients and controls were severe medical illness, neurological disorder, psychiatric disease, hearing or eyesight loss, and obvious abnormalities visible by cranial MRI. Participants who had been prescribed psychiatric drugs were also excluded.

The objectives of the research were explained to the participants and their families, and written informed consent was obtained for each participant. The research was approved by the Ethics Committee of the Beijing Tiantan Hospital.

\section{Clinical Evaluations Cognitive Assessments}

The cognitive assessments were administered by technicians according to a standard procedure and scored by a neuropsychologist. The time required for test administration was $\sim 90$ min.

The test battery included global cognitive screening, attention/processing speed, executive function, memory aptitude, language facilities, and visuospatial abilities. The MMSE and the Montreal Cognitive Assessment (MoCA) (Beijing version) (10) were used for global cognitive screening. The Digit Span Forward subset of the Wechsler Adult Intelligence TestRevised Chinese version (WAIS-RC) (11), the Trail Making Test A (TMT-A) (7), the Stroop Color-Word Test (modified version) (SCWT) Part D (7), and the Digit Symbol subtest of the WAIS-RC (11) were used to assess processing speed/attention. Executive function was assessed using the Digit Span Backward subset of the WAIS-RC, the Chinese Version of Trail Making Test B (TMT-B) (12), and the SCWT Part C (7). The RAVLT including learning measure (the sum of trials 1-5), immediate and delayed recall, and recognition, and the ROCF including immediate and delayed recall were used to detect memory (7). 
The Semantic Category Verbal Fluency Test (animal) (7) and the Boston Naming Test (BNT) as modified by Cheung et al. (13) were employed to assess language ability. Visuospatial skills were verified by the copy part of the ROCF (7), the Block Design of the WAIS-RC (11), and the Clock Drawing Test (CDT) (14) scored by the Rouleau system. The raw scores were documented in all cognitive tests.

\section{MRI}

All subjects were scanned with a standardized scan protocol on 1.5- or 3.0-Tesla MRI scanners, including T1-weighted imaging, T2-weighted imaging, diffusion weighted imaging, fluid-attenuated inversion recovery sequence (FLAIR), and susceptibility-weighted imaging (SWI). All scans were visually rated by two trained neurologists who were blinded for the diagnosis.

EPVS were defined as fluid-filled spaces with a signal intensity similar to CSF on all sequences, which followed the course of penetrating vessels. They appeared linear, round, or ovoid, with a diameter generally smaller than $3 \mathrm{~mm}$. EPVS were rated using a four-point visual rating scale on axial-T2-weighted images (1: <10 EPVS; 2: 11-20 EPVS; 3: 21-40 EPVS; and 4: >40 EPVS) for the basal ganglia (BG) and centrum semiovale (CSO) (15). The numbers of EPVS were counted in the slice with the highest number. The numbers refer to EPVS on one side of the brain, and the higher score was used if there was asymmetry between both hemispheres. The interrater reliability for the whole group was 0.753 and 0.807 for the scores of BG and CSO EPVS, respectively $(p<0.001)$.

$\mathrm{WMH}$ were hyperintense on $\mathrm{T} 2$-weighted sequences or FLAIR images. The degree of the severity was rated on FLAIR images according to the Fazekas score (range 0-3) (16). Periventricular (PV) and deep white matter hyperintensities (DWMH) were scored separately. The PV WMH score was 0 (absence), 1 (caps or pencil-thin lining), 2 (smooth halo), or 3 (irregular PV lesions extending into the deep white matter). The DWMH score was 0 (absence), 1 (punctate foci), 2 (beginning confluence of foci), or 3 (large confluent areas). The interrater reliability for the whole group was 0.771 and 0.816 for the scores of PV WMH and DWMH, respectively $(p<0.001)$.

CMBs were defined as small (generally $2-5 \mathrm{~mm}$ in diameter, but up to $10 \mathrm{~mm}$ ) areas of signal void with associated blooming seen on SWI and were generally not seen on FLAIR, T1-weighted, or T2-weighted sequences. CMBs were classified manually as lobar CMBs (suggestive of cerebral amyloid angiopathy) and deep or infratentorial CMBs (suggestive of hypertensive arteriopathy) (17). The former included different cortical regions, and the latter included the basal ganglia, thalamus, internal capsule, external capsule, corpus callosum, deep and periventricular white matter, brainstem, and cerebellum. The interrater reliability for the whole group for the presence of CMBs was $0.882(p<0.001)$.

T1-weighted or FLAIR images were used to investigate regional brain atrophy by three visual rating scales: the medial temporal lobe atrophy (MTA) scale (18), the posterior atrophy (PA) scale (19), and the global cortical atrophy scale-frontal
(GCA-F) subscale (20). The MTA was rated based on the fivepoint scale for the left or right side, respectively $(0=$ normal, $1=$ widened choroid fissure, $2=$ increase of widened fissure, widening temporal horn, opening of other sulci, $3=$ pronounced volume loss of hippocampus, $4=$ end stage atrophy). The PA was rated using the posterior cortical atrophy scale (range 03 ) with the average score of the left and right sides $(0=$ no atrophy, 1 = mild atrophy, opening of sulci, $2=$ moderate atrophy, volume loss gyri, $3=$ severe atrophy, knife blade). The GCA-F was assessed visually on the scale (range $0-3)(0=$ no atrophy, $1=$ mild atrophy, opening of sulci, $2=$ moderate atrophy, volume loss gyri, $3=$ severe atrophy, knife blade). The interrater reliability for the whole group was $0.885,0.813,0.900$, and 0.846 for the scores of GCA-F, MTA of left or right side, and $\mathrm{PA}$, respectively $(p<0.001)$.

\section{Statistical Analysis}

Statistical analyses were performed using SPSS, version 17.0 (SPSS Inc., USA). Data are expressed as the mean \pm SD unless otherwise specified. One-way analysis of variance (ANOVA) was applied for quantitative demographic variables among the control, amMCI, asMCI, nasMCI, and namMCI groups. We compared the results of the cognitive tests using analysis of covariance (ANCOVA) adjusted for age and education. Because multiple cognitive tests were administered, Bonferroni correction for multiple tests was also applied ( $p$ $<0.0025)$. The scores of brain atrophy were also assessed among all groups using ANOVA including the MTA, PA, and GCA-F.

The chi-square test was used to compare the differences between the qualitative variables among five groups, such as the sex ratio. The tests were also used to assess frequency distributions of neuroimaging variables (WMH, CMBs, and EPVS) in different MCI subgroups and healthy controls. All groups were dichotomized according to the score of PV or DMH $(\geqq 2$ or $<2$ ), lobar or deep or infratentorial CMBs (absent or present), and the scores of EPVS for BG or CSO ( $\geqq 2$ or $<2)$.

The Spearman correlation coefficient $(r)$ was used to evaluate the correlations between imaging variables, including $\mathrm{WMH}$, EPVS, MTA, PA, and GCA-F, and age, education, and scores of cognitive tests in all MCI groups. Binary logistic regression analysis was also used to assess the relationships between CMBs and age, education, and scores of cognitive tests and other imaging variables.

All statistical tests were two-tailed, and $p<0.05$ was considered to indicate statistical significance.

\section{RESULTS}

\section{Comparison of Demographic and Cognitive Data Among Groups}

The demographic and clinical data of the patients with amMCI, asMCI, namMCI, nasMCI, and the healthy controls are summarized in Table 1.

The healthy controls were younger than patients with amMCI, asMCI, and namMCI, whereas amMCI patients were slightly older than nasMCI patients. There were no 
TABLE 1 | Demographic, cognitive data of the patients with different MCl subtype and the healthy controls.

\begin{tabular}{|c|c|c|c|c|c|c|c|}
\hline Items & $\begin{array}{c}\text { amMCl } \\
(n=109)\end{array}$ & $\begin{array}{l}\text { asMCl } \\
(n=38)\end{array}$ & $\begin{array}{c}\text { namMCl } \\
(n=36)\end{array}$ & $\begin{array}{l}\text { nasMCl } \\
(n=53)\end{array}$ & $\begin{array}{l}\text { Controls } \\
(n=85)\end{array}$ & $P$ & Comparison among groups \\
\hline Age (years) & $74.52 \pm 8.25$ & $74.66 \pm 8.47$ & $73.86 \pm 10.30$ & $71.30 \pm 8.79$ & $68.64 \pm 8.62$ & $<0.001$ & $\begin{array}{l}\text { Controls < amMCl, asMCl, } \\
\text { namMCl; nasMCl < amMCl }\end{array}$ \\
\hline Gender (male \%) & 45.9 & 50.0 & 52.8 & 49.1 & 38.8 & 0.58 & \\
\hline $\begin{array}{l}\text { Education } \\
\text { (years) }\end{array}$ & $13.08 \pm 3.62$ & $14.68 \pm 2.37$ & $13.17 \pm 3.18$ & $14.43 \pm 2.92$ & $14.41 \pm 2.21$ & 0.004 & amMCl < controls, asMCl \\
\hline MMSE & $26.14 \pm 2.16$ & $27.16 \pm 1.88$ & $27.44 \pm 2.24$ & $27.70 \pm 1.71$ & $28.55 \pm 1.35$ & $<0.001$ & amMCl < controls; nasMCl \\
\hline MoCA & $21.23 \pm 3.27$ & $23.76 \pm 2.55$ & $22.59 \pm 3.56$ & $24.37 \pm 2.61$ & $25.80 \pm 2.28$ & $<0.001$ & $\begin{array}{c}\text { amMCl, namMCl < controls; } \\
\text { amMCl < asMCl, nasMCl }\end{array}$ \\
\hline $\begin{array}{l}\text { Digit Span } \\
\text { Forward }\end{array}$ & $7.37 \pm 1.14$ & $8.00 \pm 0.66$ & $6.89 \pm 1.30$ & $7.34 \pm 1.47$ & $8.20 \pm 0.86$ & $<0.001$ & $\begin{array}{c}\text { namMCl, nasMCl }<\text { controls; } \\
\text { namMCl }<\text { asMCl }\end{array}$ \\
\hline TMT-A & $86.35 \pm 37.12$ & $58.32 \pm 18.83$ & $83.17 \pm 29.86$ & $65.36 \pm 24.74$ & $\begin{array}{l}49.19 \pm \\
14.77\end{array}$ & $<0.001$ & $\begin{array}{c}\text { Controls }<\text { amMCl, namMCl; } \\
\text { asMCl }<\text { amMCl }\end{array}$ \\
\hline SCWT Part D & $20.23 \pm 7.70$ & $15.49 \pm 4.14$ & $19.69 \pm 6.10$ & $17.85 \pm 5.61$ & $14.05 \pm 3.32$ & $<0.001$ & Controls < amMCl, namMCl \\
\hline Digit Symbol & $26.06 \pm 8.08$ & $36.16 \pm 9.76$ & $27.33 \pm 7.65$ & $33.15 \pm 8.72$ & $39.41 \pm 9.59$ & $<0.001$ & $\begin{array}{c}\text { amMCl, namMCl, nasMCl }< \\
\text { controls; amMCl, namMCl }< \\
\text { asMCl }\end{array}$ \\
\hline $\begin{array}{l}\text { Digit Span } \\
\text { Backward }\end{array}$ & $4.18 \pm 1.14$ & $4.92 \pm 0.94$ & $4.11 \pm 0.92$ & $4.66 \pm 1.22$ & $5.40 \pm 1.30$ & $<0.001$ & amMCl, namMCl < controls \\
\hline TMT-B & $\begin{array}{c}233.07 \pm \\
132.20\end{array}$ & $116.47 \pm 42.89$ & $\begin{array}{c}205.31 \pm \\
100.28\end{array}$ & $141.55 \pm 62.28$ & $\begin{array}{c}93.25 \pm \\
38.20\end{array}$ & $<0.001$ & $\begin{array}{l}\text { Controls, asMCl < amMCl, } \\
\text { namMCl; nasMCl < amMCl }\end{array}$ \\
\hline SCWT Part C & $40.86 \pm 14.74$ & $31.43 \pm 6.27$ & $37.57 \pm 13.47$ & $33.77 \pm 9.82$ & $28.39 \pm 7.36$ & $<0.001$ & Controls, asMCl < amMCl \\
\hline $\begin{array}{l}\text { Learning } \\
\text { measure of } \\
\text { RAVLT }\end{array}$ & $27.64 \pm 7.90$ & $29.63 \pm 8.27$ & $39.64 \pm 7.08$ & $40.51 \pm 7.80$ & $42.73 \pm 8.18$ & $<0.001$ & $\begin{array}{c}\text { amMCl, asMCl < controls, } \\
\text { namMCl, nasMCl }\end{array}$ \\
\hline $\begin{array}{l}\text { Immediate recall } \\
\text { of RAVLT }\end{array}$ & $4.43 \pm 2.65$ & $5.18 \pm 3.10$ & $8.56 \pm 2.10$ & $8.53 \pm 2.42$ & $9.05 \pm 2.27$ & $<0.001$ & $\begin{array}{c}\text { amMCl, asMCl < controls, } \\
\text { namMCl, nasMCl }\end{array}$ \\
\hline $\begin{array}{l}\text { Delayed recall of } \\
\text { RAVLT }\end{array}$ & $3.03 \pm 2.88$ & $3.89 \pm 2.66$ & $7.31 \pm 2.14$ & $7.89 \pm 2.69$ & $8.20 \pm 2.57$ & $<0.001$ & $\begin{array}{c}\text { amMCl, asMCl < controls, } \\
\text { namMCl, nasMCl }\end{array}$ \\
\hline $\begin{array}{l}\text { Recognition of } \\
\text { RAVLT }\end{array}$ & $8.37 \pm 3.80$ & $8.97 \pm 2.84$ & $13.39 \pm 1.50$ & $13.06 \pm 1.61$ & $13.38 \pm 1.40$ & $<0.001$ & $\begin{array}{c}\text { amMCl, asMCl < controls, } \\
\text { namMCl, nasMCl }\end{array}$ \\
\hline $\begin{array}{l}\text { Immediate recall } \\
\text { of } \mathrm{ROCF}\end{array}$ & $11.09 \pm 7.09$ & $14.03 \pm 7.38$ & $19.30 \pm 4.22$ & $21.14 \pm 4.55$ & $23.48 \pm 5.16$ & $<0.001$ & $\begin{array}{c}\text { amMCl, asMCl < controls, } \\
\text { namMCl, nasMCl }\end{array}$ \\
\hline $\begin{array}{l}\text { Delayed recall of } \\
\text { ROCF }\end{array}$ & $9.31 \pm 7.40$ & $13.33 \pm 7.11$ & $19.64 \pm 3.63$ & $20.34 \pm 4.16$ & $22.79 \pm 5.01$ & $<0.001$ & $\begin{array}{c}\text { amMCl, asMCl < controls, } \\
\text { namMCl, nasMCl }\end{array}$ \\
\hline Verbal fluency & $14.40 \pm 3.85$ & $17.95 \pm 3.06$ & $14.97 \pm 4.50$ & $17.79 \pm 3.76$ & $18.96 \pm 3.42$ & $<0.001$ & $\begin{array}{c}\text { amMCl, namMCl < control; } \\
\text { amMCl < asMCl, nasMCl }\end{array}$ \\
\hline BNT & $21.10 \pm 3.62$ & $24.47 \pm 2.40$ & $20.94 \pm 3.55$ & $24.02 \pm 3.82$ & $25.08 \pm 2.39$ & $<0.001$ & $\begin{array}{c}\text { amMCl, namMCl }<\text { controls, } \\
\text { asMCl, nasMCl }\end{array}$ \\
\hline $\begin{array}{l}\text { Copy part of } \\
\text { ROCF }\end{array}$ & $32.63 \pm 3.81$ & $34.33 \pm 1.89$ & $33.78 \pm 2.39$ & $33.85 \pm 3.20$ & $34.64 \pm 1.66$ & $<0.001$ & amMCl $<$ controls \\
\hline Block design & $24.39 \pm 6.67$ & $29.42 \pm 6.15$ & $26.19 \pm 7.00$ & $29.49 \pm 6.45$ & $33.14 \pm 6.74$ & $<0.001$ & amMCl, namMCl, < controls \\
\hline CDT & $8.19 \pm 2.01$ & $9.47 \pm 0.80$ & $7.86 \pm 2.49$ & $9.00 \pm 1.44$ & $9.61 \pm 0.68$ & $<0.001$ & $\begin{array}{c}\text { amMCl, namMCl }<\text { control, } \\
\text { asMCl }\end{array}$ \\
\hline
\end{tabular}

differences in age among other groups. Patients with amMCI had lower education levels than patients with asMCI and healthy controls, but there were no differences in education among other groups. There were no sex differences among different groups.

After adjusting age and education, patients with amMCI performed worse than patients with nasMCI and healthy controls on the MMSE (Bonferroni correction was applied and level of significance set at 0.0025 ). The scores of the MoCA in patients with amMCI and namMCI were lower than those of healthy controls, whereas patients with amMCI also had worse scores than patients with asMCI and nasMCI.

On the attention/processing speed domain, the Digit Span Forward scores in patients with namMCI and nasMCI were lower than those of healthy controls, while patients with namMCI also performed worse than patients with asMCI. Patients with amMCI and namMCI performed worse on the TMT-A and the SCWT Part D than healthy controls, whereas patients with amMCI spent a longer time on the TMT-A compared with patients with asMCI. Patients with amMCI, namMCI, and nasMCI had lower scores 
of the Digit Symbol than healthy controls, while patients with amMCI and namMCI did worse than patients with asMCI.

On the domain of executive function, the scores of patients with amMCI and namMCI were lower on the Digit Span Backward than those of healthy controls. Patients with amMCI and namMCI performed worse on the TMT-B than healthy controls and patients with asMCI, while the amMCI group also performed worse than the nasMCI group. As to SCWT Part C, healthy controls and patients with asMCI spent a shorter time than did patients with amMCI.

On the domain of memory, patients with amMCI and asMCI had lower scores than patients with namMCI and nasMCI and healthy controls on the learning measure, immediate and delayed recall, and recognition of the RAVLT. The scores of patients with amMCI and asMCI were also lower than those of patients with namMCI and nasMCI and healthy scores on immediate and delayed recall of the ROCF.

$5 \mathrm{On}$ the domain of language facilities, patients with amMCI and namMCI had lower scores than patients with asMCI and nasMCI and healthy controls on the BNT. Patients with amMCI and namMCI performed worse than healthy controls on the verbal fluency test, while the scores of patients with amMCI were lower than those of patients with asMCI, nasMCI.

On the domain of visuospatial abilities, patients with amMCI and namMCI had lower scores on the Block Design than healthy controls. Patients with amMCI and namMCI performed worse than patients with asMCI and healthy controls on the CDT. As to the copy part of the ROCF, the scores of patients with amMCI were lower than those of healthy controls.

\section{Comparison of Imaging Data of CSVD Among Groups}

We compared the percentages of participants with higher scores of PV WMH or DWMH, lobar or deep or infratentorial CMBs, higher scores of EPVS for BG or CSO, and the degree of brain atrophy represented as scores of MTA, PA, and GCA-F among groups.

The patients with amMCI, namMCI, and nasMCI had more high-grade BG EPVS compared with healthy controls, while the percentages of high-grade BG EPVS in the patients with amMCI were also more than those in patients with asMCI, namMCI, and nasMCI. There were more high-grade CSO EPVS in patients with amMCI in comparison with all other groups (Table 2, Figure 1).

The patients with amMCI and namMCI had more severe DWMH compared with healthy controls, while patients with amMCI also had higher percentages of severe DWMH than patients with asMCI. There were marginal differences between amMCI and nasMCI $(p=0.061)$ and asMCI and namMCI $(p$ $=0.093)$. Patients of amMCI and namMCI had more severe PVWMH than healthy controls, whereas the namMCI group also had higher percentages of severe PVWMH compared with the asMCI and nasMCI groups, although the differences were marginal ( $p=0.065,0.051)$ (Table 2, Figure 1).

Patients with MCI had 19.1\% (45/236) deep or infratentorial and $13.1 \%(31 / 236)$ lobe CMBs. Patients with amMCI and namMCI had more deep or infratentorial CMBs than healthy controls, whereas there were also more deep or infratentorial CMBs in patients with amMCI compared with patients with asMCI and nasMCI. The differences between asMCI and nasMCI patients and healthy controls were marginal ( $p=0.052,0.065)$. As to lobar CMBs, there were no differences among groups (Table 2, Figure 1).

All MCI groups had higher total scores of the MTA than healthy controls, whereas the scores of the amMCI group were also higher than those of the namMCI and nasMCI groups. Patients with amMCI, asMCI, and nasMCI had higher scores of left and right MTA than healthy controls, while there were higher scores of right MTA on patients with amMCI compared with patients with namMCI. Patients with amMCI had higher scores of GCA-F than healthy controls. There were high scores of PA on patients with amMCI, asMCI, and namMCI compared with healthy controls (Table 2, Figure 2).

\section{Correlations Between Variables of CSVD and Cognitive Tests}

In MCI groups, BG EPVS had positive correlations with white matter hyperintensities and frontal and posterior atrophy in addition to CSO EPVS, while there were correlations between BG EPVS and the MoCA, and tests of cognitive domains including attention/processing speed, executive function, verbal memory, language facilities, and visuospatial abilities. CSO EPVS had positive correlations with white matter hyperintensities, while there were only correlations between CSO EPVS and learning measures of RAVLT, the verbal fluency, and the SCWT Part D.

PVWMH had positive correlations with medial temporal lobe atrophy in addition to DWMH, whereas DWMH had positive correlations with medial temporal lobe and posterior atrophy. There were correlations between WMH and age and tests involving attention/processing speed and executive function.

Frontal atrophy had positive correlations with medial temporal lobe and posterior atrophy, whereas there was a positive relationship between medial temporal lobe atrophy and posterior atrophy. Frontal atrophy had correlations with age, education, the MMSE, and tests including attention/processing speed, executive function, and verbal and visuospatial memory. Medial temporal lobe atrophy also had correlations with age, education, the MMSE, and tests including attention/processing speed, executive function, and verbal and visuospatial memory. Posterior atrophy only had correlations with age, education, and tests including attention/processing speed, executive function, and learning measures of RAVLT (Table 3).

Binary logistic regression analysis showed that only age contributed to lobar CMBs (OR = 1.160, 95 CI 1.035-1.301, $p=$ 0.011 ). There was no relationship between deep or infratentorial CMBs and any other imaging and cognitive indicators.

\section{DISCUSSION}

The present study investigated cognitive and imaging features of CSVD in MCI. There were obvious cognitive impairments in different MCI groups, especially amMCI. Different imaging changes of CSVD were also shown in various MCI patients. 
TABLE 2 | Imaging data of the patients with different MCl subtypes and the healthy controls.

\begin{tabular}{|c|c|c|c|c|c|c|c|}
\hline Items & $\begin{array}{c}\text { amMCl } \\
(n=109)\end{array}$ & $\begin{array}{l}\text { asMCl } \\
(n=38)\end{array}$ & $\begin{array}{l}\text { namMCl } \\
(n=36)\end{array}$ & $\begin{array}{l}\text { nasMCl } \\
(n=53)\end{array}$ & $\begin{array}{l}\text { Controls } \\
(n=85)\end{array}$ & $\boldsymbol{P}$ & Comparison among groups \\
\hline BG EPVS (\%) & 79.8 & 31.6 & 47.2 & 47.2 & 22.4 & $<0.001$ & $\begin{array}{c}\text { Controls < amMCl, namMCl, } \\
\text { nasMCl; asMCl, namMCl, } \\
\text { nasMCl < amMCl }\end{array}$ \\
\hline CSO EPVS (\%) & 49.5 & 28.9 & 27.8 & 24.5 & 27.1 & 0.002 & $\begin{array}{c}\text { Controls, asMCl, namMCl, } \\
\text { nasMCl }<\text { amMCl }\end{array}$ \\
\hline PVWMH (\%) & 41.3 & 31.6 & 52.8 & 32.1 & 20.0 & 0.003 & Controls < amMCl, namMCl \\
\hline DWMH (\%) & 39.4 & 21.1 & 38.9 & 24.5 & 14.1 & 0.001 & $\begin{array}{c}\text { Controls }<\text { amMCl, namMCl; } \\
\text { asMCl }<\text { amMCl }\end{array}$ \\
\hline Lobar CMBs (\%) & 15.6 & 5.3 & 19.4 & 9.4 & 21.2 & 0.129 & \\
\hline $\begin{array}{l}\text { Deep or } \\
\text { infratentorial } \\
\text { CMBs (\%) }\end{array}$ & 26.6 & 10.5 & 19.4 & 9.4 & 2.4 & $<0.001$ & $\begin{array}{c}\text { Controls < amMCl, namMCl; } \\
\text { asMCl, nasMCl < amMCl }\end{array}$ \\
\hline GCA-F & $1.35 \pm 0.53$ & $1.36 \pm 0.55$ & $1.31 \pm 0.47$ & $1.18 \pm 0.39$ & $1.05 \pm 0.45$ & 0.002 & Control $<$ amMCl \\
\hline MTA & $3.53 \pm 1.36$ & $3.36 \pm 1.43$ & $2.88 \pm 1.37$ & $3.00 \pm 1.16$ & $2.13 \pm 1.34$ & $<0.001$ & $\begin{array}{c}\text { Controls < amMCl, asMCl, } \\
\text { namMCl, nasMCl; namMCl, } \\
\text { nasMCl }<\text { amMCl }\end{array}$ \\
\hline Left MTA & $1.73 \pm 0.74$ & $1.67 \pm 0.82$ & $1.46 \pm 0.71$ & $1.45 \pm 0.60$ & $1.00 \pm 0.69$ & $<0.001$ & $\begin{array}{c}\text { Controls }<\text { amMCl, asMCl, } \\
\text { nasMCl }\end{array}$ \\
\hline Right MTA & $1.80 \pm 0.69$ & $1.70 \pm 0.68$ & $1.42 \pm 0.70$ & $1.55 \pm 0.60$ & $1.13 \pm 0.70$ & $<0.001$ & $\begin{array}{c}\text { Controls }<\text { amMCl, asMCl, } \\
\text { namMCl, nasMCl; namMCl }< \\
\text { amMCl }\end{array}$ \\
\hline PA & $1.45 \pm 0.57$ & $1.48 \pm 0.67$ & $1.58 \pm 0.58$ & $1.38 \pm 0.59$ & $1.22 \pm 0.60$ & $<0.001$ & $\begin{array}{c}\text { Controls }<\text { amMCl, asMCl, } \\
\text { namMCl }\end{array}$ \\
\hline
\end{tabular}

\section{comparison of EPVS, WMH, CMBs among groups}

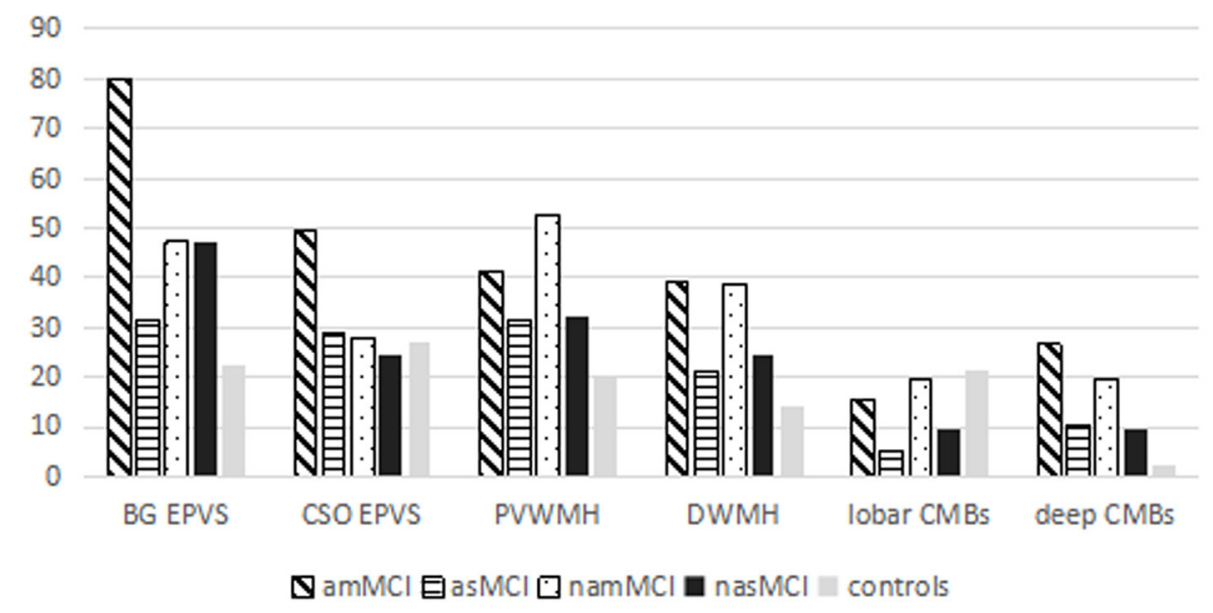

FIGURE 1 | Comparison of EPVS, WMH, and CMBs among all groups.

Patients with aMCI had obvious memory impairments than naMCI and controls on all memory tests. There were heterogeneous results on different tests of other domains. The multiple-domain MCI group performed worse on executive function and visuospatial abilities compared with controls. Patients with amMCI and naMCI had worse scores than controls on the attention domain, while patients with multiple-domain MCI performed worse on the language domain compared with single-domain MCI and controls.

The patients with amMCI had more high-grade BG PVS followed by patients with namMCI and nasMCI compared with healthy controls, while there were more high-grade CSO PVS in patients with amMCI in comparison with all other groups. EPVS had obvious correlations with PV WMH and DWMH, whereas BG EPVS also had relationships with frontal and parietal atrophy. 


\section{comparison of cerebral atrophy among groups}

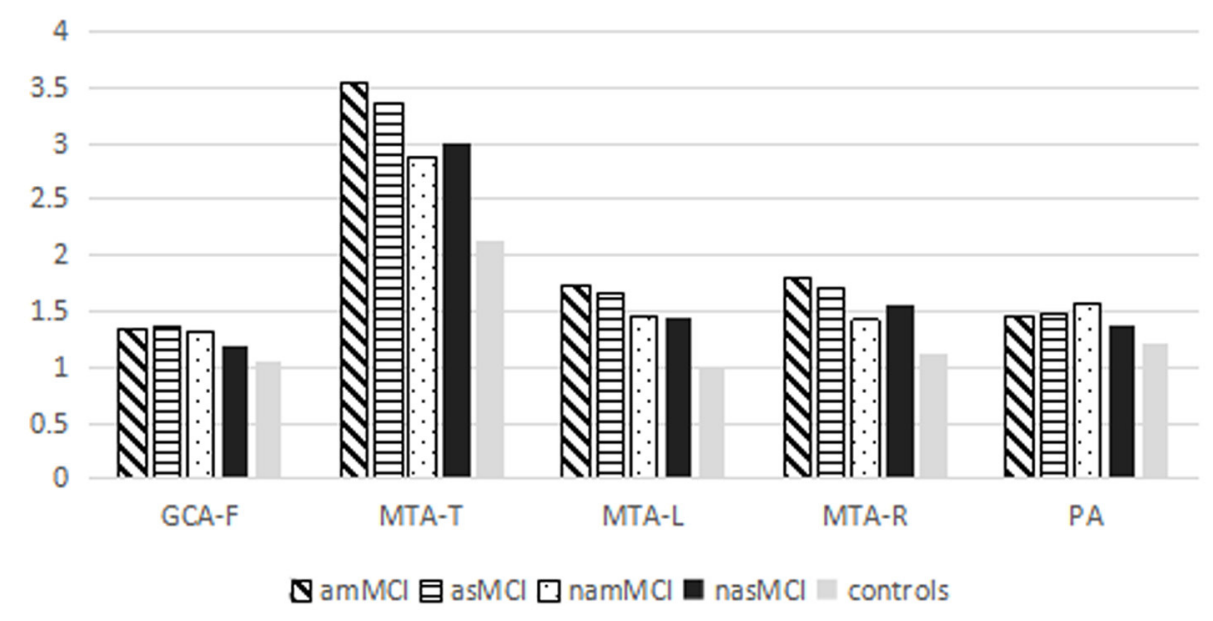

FIGURE 2 | Comparison of cerebral atrophy among all groups.

There was a correlation between BG EPVS and every domain of cognition tests, but COS EPVS had only a relationship with attention, language, and learning parts of the verbal memory test.

Perivascular spaces are extensions of the extracerebral fluid space around arteries, arterioles, veins, and venules, which are the main drainage conduits and form part of the glymphatic system (4). EPVS could involve impairment of cerebrovascular reactivity, blood-brain barrier dysfunction, perivascular inflammation, and abnormal clearance of waste proteins from the interstitial fluid space, ultimately leading to accumulation of toxins, hypoxia, and tissue damage (21). All pathophysiological processes could increase WMH, affect clearance of $\beta$ amyloid, and cause its accumulation in the brain, which increase the risk of cognitive decline and dementia and play a potential key role on the pathogenesis of AD. Previous studies found that EPVS was associated with WMH but not atrophy $(4,22)$. We demonstrated it in patients with MCI, but the relationship between BG EPVS and frontal and parietal atrophy was also reported. We found that EPVS of various regions had different effects on cognition and MCI. Patients with amMCI had more high-grade BG and CSO EPVS, whereas there was more high-grade BG EPVS in only naMCI patients. A Spanish study reported that BG EPVS not CSO could predict MCI in hypertensive individuals although affected by other markers of CSVD (23). The scores of BG EPVS were higher in vascular dementia than those of $\mathrm{AD}$, and there were no differences on CSO EPVS scores (24). Another study showed that a high degree of white matter EPVS is associated with the number of lobar MBs, while a high degree of BG EPVS is associated with the presence of hypertension. The results meant that white matter EPVS had a relationship with cerebral amyloid angiopathy (CAA) (25). CSO EPVS might indicate the presence of CAA or a mixed hypertensive/CAA. However, we found no correlation between EPVS and CMBs, which may contribute the method assessing
CMBs. Participants with severe EPVS in both regions or in the CSO alone had greater decline in global cognition, and the presence of severe EPVS in both regions was an independent predictor of dementia. The study did not find the association between cognitive domain and EPVS (26). Our study found that BG EPVS had a relationship with more extensive cognitive domain compared with CSO EPVS.

The patients with amMCI and namMCI had more percentages of severe DWMH and PVWMH compared with healthy controls. There were more severe DWMH in patients with amMCI compared with asMCI. There were marginal differences between amMCI and nasMCI and namMCI and asMCI in DWMH, and namMCI and asMCI or nasMCI in PVWMH. WMH had a correlation with attention, executive function, and medial temporal lobe atrophy.

The pathogenesis of WHM is not well-understood and could be multifactorial, although strongly associated with cerebrovascular disease and vascular risk factors (4). In the baseline cognitively normal individuals, greater $\mathrm{WMH}$ were associated with accelerated multiple-domain cognitive, neuropsychiatric, and functional decline independent of traditional risk factors. WMH also contributed to the development of MCI $(27,28)$. There was no statistical difference within various MCI types regarding $\mathrm{DWMH}$, while PVWMH scores were significantly higher in patients with naMCI than those in patients with aMCI (29). The aMCI group showed elevated temporal and occipital $\mathrm{WMH}$ volume relative to the control group whereas the naMCI group showed elevated WMH volume across frontal, parietal, temporal, and occipital regions, suggesting more widespread $\mathrm{WMH}$ accumulation. In addition, the naMCI participants showed greater occipital WMH relative to the aMCI (30). Our study demonstrated that the percentages of high-grade PVWMH and DWMH were higher 
TABLE 3 | Correlation between imaging variables and age, education, and scores of cognitive tests in $\mathrm{MCl}$ groups.

\begin{tabular}{|c|c|c|c|c|c|c|c|}
\hline Items & BG EPVS & CSO EPVS & PV WMH & DWMH & GCA-F & MTA & PA \\
\hline Age & 0.122 & 0.109 & $0.276^{a}$ & $0.211^{\mathrm{a}}$ & $0.465^{a}$ & $0.455^{a}$ & $0.452^{\mathrm{a}}$ \\
\hline Education & -0.092 & 0.015 & 0.131 & 0.083 & $0.228^{a}$ & $0.234^{a}$ & $0.201^{a}$ \\
\hline BG EPVS & & $0.362^{a}$ & $0.232^{a}$ & $0.260^{a}$ & $0.224^{a}$ & 0.115 & $0.212^{a}$ \\
\hline CSO EPVS & $0.362^{a}$ & & $0.159^{a}$ & $0.239^{a}$ & -0.006 & 0.005 & 0.098 \\
\hline PV WMH & $0.232^{\mathrm{a}}$ & $0.159^{a}$ & & $0.783^{a}$ & 0.085 & $0.194^{a}$ & 0.117 \\
\hline DWMH & $0.260^{\mathrm{a}}$ & $0.239^{a}$ & $0.783^{a}$ & & 0.046 & $0.178^{a}$ & $0.147^{a}$ \\
\hline GCA-F & $0.224^{a}$ & -0.006 & 0.085 & 0.046 & & $0.312^{a}$ & $0.661^{a}$ \\
\hline MTA & 0.115 & 0.005 & $0.194^{a}$ & $0.178^{a}$ & $0.312^{a}$ & & $0.232^{a}$ \\
\hline PA & $0.212^{\mathrm{a}}$ & 0.098 & 0.117 & $0.147^{a}$ & $0.661^{a}$ & $0.232^{a}$ & \\
\hline MMSE & -0.024 & -0.033 & -0.097 & -0.091 & $-0.152^{a}$ & $-0.148^{a}$ & -0.121 \\
\hline MoCA & $-0.179^{a}$ & -0.037 & -0.073 & $-0.162^{a}$ & 0.123 & -0.065 & -0.066 \\
\hline Digit span forward & 0.022 & 0.010 & -0.050 & -0.018 & 0.043 & -0.136 & 0.026 \\
\hline TMT-A & $0.181^{a}$ & 0.083 & $0.203^{a}$ & $0.175^{a}$ & 0.142 & $0.207^{a}$ & 0.104 \\
\hline SCWT part D & $0.230^{\mathrm{a}}$ & $0.183^{a}$ & 0.116 & 0.121 & 0.113 & 0.101 & $0.148^{a}$ \\
\hline Digit symbol & $-0.208^{a}$ & -0.064 & $-0.186^{a}$ & $-0.194^{a}$ & $-0.173^{a}$ & $-0.199^{a}$ & $-0.148^{a}$ \\
\hline Digit span backward & -0.017 & -0.004 & -0.114 & -0.082 & 0.011 & 0.012 & -0.003 \\
\hline TMT-B & $0.219^{a}$ & 0.045 & $0.177^{a}$ & $0.243^{a}$ & $0.207^{a}$ & $0.160^{a}$ & $0.184^{a}$ \\
\hline SCWT part C & 0.107 & 0.044 & 0.122 & 0.100 & 0.015 & $0.149^{a}$ & 0.054 \\
\hline Learning measure of RAVLT & $-0.199^{a}$ & $-0.222^{a}$ & -0.073 & -0.092 & $-0.236^{a}$ & $-0.252^{a}$ & $-0.174^{a}$ \\
\hline Immediate recall of RAVLT & -0.145 & -0.119 & -0.015 & -0.044 & $-0.164^{a}$ & $-0.185^{b}$ & -0.111 \\
\hline Delayed recall of RAVLT & $-0.190^{a}$ & -0.124 & -0.092 & -0.102 & $-0.157^{a}$ & $-0.219^{a}$ & -0.091 \\
\hline Recognition of RAVLT & -0.125 & -0.138 & 0.063 & 0.004 & $-0.188^{a}$ & -0.123 & -0.084 \\
\hline Immediate recall of ROCF & -0.101 & -0.150 & -0.005 & -0.091 & $-0.155^{\mathrm{a}}$ & $-0.181^{a}$ & -0.026 \\
\hline Delayed recall of ROCF & -0.106 & -0.136 & 0.036 & -0.044 & $-0.159^{a}$ & $-0.202^{a}$ & -0.027 \\
\hline Verbal fluency & -0.148 & $-0.155^{a}$ & -0.045 & $-0.155^{a}$ & -0.010 & -0.047 & -0.029 \\
\hline BNT & $-0.263^{a}$ & -0.095 & -0.069 & -0.037 & -0.047 & 0.054 & -0.076 \\
\hline Copy part of ROCF & $-0.163^{a}$ & -0.077 & 0.035 & -0.051 & -0.091 & -0.008 & -0.105 \\
\hline Block design & -0.153 & 0.001 & -0.075 & -0.056 & -0.046 & -0.050 & 0.026 \\
\hline CDT & -0.068 & $0.158^{\mathrm{a}}$ & -0.070 & -0.081 & 0.036 & -0.031 & 0.121 \\
\hline
\end{tabular}

${ }^{a} p<0.05$.

in multiple-domain MCI compared with single-domain MCI or healthy controls.

The meta-analysis showed that the association between $\mathrm{WMH}$ and overall cognition was significantly stronger for MCI than for $\mathrm{AD}$ (31). For both groups, the largest effect sizes were found in attention and executive functions and processing speed. Interestingly, there was also a significant association with the memory domain which is more closely related to $\mathrm{AD}$. The study also suggested that PV WMH were more strongly related to cognition than DWMH. DWMH related to ischemic risk factors may predominantly disrupt the short association fibers, which linked to adjacent gyri. PVWMH linked to atrophic processes was likely to affect the long association fibers that connected the more distant cortical areas. The study involving executive functions revealed the association between PVWMH and working memory, DWMH and inhibition performance, and MTA and flexibility performance (32). The relationship between WMH and attention and executive function was also demonstrated in our study.
The MRI study showed that hippocampal subfield atrophy worsened with increasing CSVD severity, mainly WMH. Greater atrophy was seen with moderate to severe CSVD compared to mild CSVD in the subfields including the subiculum, CA1, CA4, molecular layer, and dentate gyrus. Atrophy in the subfields was significantly associated with poor episodic memory and frontal executive function (33). The present study also certified that WMH had an obvious correlation with MTA.

Patients with amMCI and namMCI had more deep or infratentorial $\mathrm{CMBs}$ than healthy controls, whereas there were also more deep or infratentorial CMBs in patients with amMCI compared with patients with asMCI and nasMCI. The differences between asMCI and nasMCI patients and healthy controls were marginal. As to lobar $\mathrm{CMBs}$, there were no differences among groups.

CMBs may represent hemosiderin-laden macrophages in perivascular tissue, secondary to vascular leakage of blood cells. Deep or infratentorial CMBs were hypothesized to be associated with hypertensive microangiopathy, while lobar CMBs may be 
due to CAA (4). The mechanisms of the association between CMBs and cognitive dysfunction were not well-understood.

Patients with $\mathrm{AD}$ and progress subtype of MCI had significantly more new $\mathrm{CMB}$ than controls and patients with a stable subtype of MCI during the follow-up (34). Total number of $\mathrm{CMBs}$ and of those in deep and lobar regions were associated with attention and executive function and fluency domains (35). The presence of any CMBs, including lobar and deep or infratentorial CMBs, was related to MCI after adjusting for confounders. Furthermore, the presence of multiple microbleeds is associated with lower MoCA total scores and with worse performance on specific domains of cognitive tests, such as global cognitive function, information processing speed, and motor speed (36). Our patients with MCI had $19.1 \%$ deep or infratentorial and $13.1 \%$ lobe CMBs, which was similar to a previous study (17). There were more deep or infratentorial CMBs in various MCI groups, while those in the amMCI group was also more obvious than the single-domain MCI group. There was no difference in lobe CMBs, which may contribute to high percentages of lobe CMBs in healthy controls selected from memory clinics. We did not find the association between CMBs and cognition, which could be caused by the method evaluating CMBs only absent or present.

Although brain atrophy was attributed to neurodegenerative diseases, many imaging studies reported an association between the presence and severity of SVD and brain atrophy, even including hippocampal atrophy. Moreover, atrophy was an important measure in imaging studies that were done to assess the burden of vascular damage in the brain and cognition (4). Brain atrophy was present in all MCI types and was greater in multiple-domain types particularly in the naMCI $(29,37)$. The present study found that all MCI groups had obvious atrophy of the medial temporal lobe compared with healthy controls, whereas the difference was also obvious in the amMCI group than the naMCI group. Patients with amMCI had obvious frontal atrophy, while there was a prominent parietal atrophy in patients with aMCI and namMCI in comparison with healthy controls. Episodic memory is often considered as a marker of early stage in patients with AD. Scores of episodic memory tests were reported to correlate with hippocampal volumes in MCI, which also were associated with executive function $(33,38)$. Our study showed that frontal and medial temporal lobe atrophy had an obvious correlation with attention, executive function, and learning and memory, whereas there was a relationship between parietal atrophy and attention, executive function and learning part of verbal memory.

\section{REFERENCES}

1. Jia L, Du Y, Chu L, Zhang Z, Li F, Lyu D, et al. Prevalence, risk factors, and management of dementia and mild cognitive impairment in adults aged 60 years or older in China: a cross-sectional study. Lancet Public Health. (2020) 5:e661-71. doi: 10.1016/S2468-2667(20)30185-7

2. Petersen RC, Doody R, Kurz A, Mohs RC, Morris JC, Rabins PV, et al. Current concepts in mild cognitive impairment. Arch Neurol. (2001) 58:198592. doi: 10.1001/archneur.58.12.1985
The present study has some limitations. First, the participants were selected from our memory clinics, not from the community, which may lead to bias. Second, more patients with amMCI were selected compared with asMCI and namMCI. Thirdly, the healthy controls were younger, and patients with amMCI had lower education levels, but we analyzed the results after adjusting the age and education.

In conclusion, patients with different MCI types had obvious cognitive impairments, especially amMCI. The imaging of CSVD with different frequencies, such as EPVS, WMH, CMBs, and brain atrophy, was found in various MCI groups. There was a relationship between varied neuroimaging features of CSVD and cognitive impairment. All of those meant that the vascular mechanism contributed to the prodromal stage of dementia.

\section{DATA AVAILABILITY STATEMENT}

The raw data supporting the conclusions of this article will be made available by the authors, without undue reservation.

\section{ETHICS STATEMENT}

The studies involving human participants were reviewed and approved by the Ethics Committee of the Beijing Tiantan Hospital. The patients/participants provided their written informed consent to participate in this study.

\section{AUTHOR CONTRIBUTIONS}

$\mathrm{XL}, \mathrm{XT}$, and JJ contributed to the conception and design of the study. XL analyzed and interpreted the data. XL and $\mathrm{XT}$ revised the manuscript. $\mathrm{MS}, \mathrm{YJ}, \mathrm{SJ}, \mathrm{ZZ}, \mathrm{ZH}$, and $\mathrm{XZ}$ contributed to the participants' enrollment and the clinical assessments. MS wrote the first draft of the manuscript. All the authors contributed to the article and approved the submitted version.

\section{FUNDING}

This work was supported by Key Projects of Strategic International Cooperation in Scientific and Technological Innovation in the National Key Research and Development Program (2018YFE0203600), Key Area in Research and Development Program of Guangdong Province (2018B030336001), and personnel development funding projects of Beijing Dongcheng District (DCQYYRC-791-01-DR).

3. Petersen RC, Caracciolo B, Brayne C, Gauthier S, Jelic V, Fratiglioni L. Mild cognitive impairment: a concept in evolution. J Intern Med. (2014) 275:214-28. doi: 10.1111/joim. 12190

4. Wardlaw JM, Smith EE, Biessels GJ, Cordonnier C, Fazekas F, Frayne F, et al. Neuroimaging standards for research into small vessel disease and its contribution to ageing and neurodegeneration. Lancet Neurol. (2013) 12:822-38. doi: 10.1016/S1474-4422(13)7 0124-8 
5. Wardlaw JM, Smith C, Dichgans M. Small vessel disease: mechanisms and clinical implications. Lancet Neurol. (2019) 18:684-96. doi: 10.1016/S1474-4422(19)30079-1

6. Ter Telgte A, van Leijsen EMC, Wiegertjes K, Klijn CJM, Tuladhar AM, de Leeuw FE. Cerebral small vessel disease: from a focal to a global perspective. Nat Rev Neurol. (2018) 14:387-98. doi: 10.1038/s41582-018-0014-y

7. Strauss E, Sherman EM, Spreen O. A Compendium of Neuropsychological Tests: Administration, Norms, and Commentary. 3rd ed. New York, NY: Oxford University Press (2006).

8. Zhang MY, Katzman R, Salmon D, Jin H, Cai GJ, Wang ZY, et al. The prevalence of dementia and Alzheimer's disease in Shanghai, China: impact of age, gender, and education. Ann Neurol. (1990) 27:42837. doi: 10.1002/ana.410270412

9. Morris JC. The clinical dementia rating (CDR): current version and scoring rules. Neurology. (1993) 43:2412-4. doi: 10.1212/WNL.43.11.2412-a

10. Nasreddine Z, Phillips NA, Bedirian V, Charbonneau S, Whitehead V, Collin I, et al. The montreal cognitive assessment, MoCA: a brief screening tool for mild cognitive impairment. J Am Geriatr Soc. (2005) 53:6959. doi: 10.1111/j.1532-5415.2005.53221.x

11. Dai XY, Ryan JJ, Paolo AM, Harrington RG. Factor analysis of the mainland Chinese version of the Wechsler Adult Intelligence Scale (WAIS-RC) in a brain-damaged sample. Int J Neurosci. (1990) 55:107-11. doi: 10.3109/002074590089 85956

12. Lu L, Bigler ED. Performance on original and a Chinese version of trail making test part B: a normative bilingual sample. Appl Neuropsychol. (2000) 7:243-46. doi: 10.1207/S15324826AN07 $04-6$

13. Cheung RW, Cheung MC, Chan AS. Confrontation naming in Chinese patients with left, right or bilateral brain damage. $J$ Int Neuropsychol Soc. (2004) 10:46-53. doi: 10.1017/S13556177041 01069

14. Rouleau I, Salmon DP, Butters N, Kennedy C, McGuire K. Quantitative and qualitative analyses of clock drawings in Alzheimer's and Huntington's disease. Brain cogn. (1992) 18:70-87. doi: 10.1016/0278-2626(92)90 $112-\mathrm{Y}$

15. Potter GM, Chappell FM, Morris Z, Wardlaw JM. Cerebral perivascular spaces visible on magnetic resonance imaging: development of a qualitative rating scale and its observer reliability. Cerebrovasc Dis. (2015) 39:224-31. doi: 10.1159/0003 75153

16. Fazekas F, Chawluk JB, Alavi A, Hurtig HI, Zimmerman RA. MR signal abnormalities at $1.5 \mathrm{~T}$ in Alzheimer's dementia and normal aging. Am J Roentgenol. (1987) 149:351-6. doi: 10.2214/ajr.149. 2.351

17. Greenberg SM, Vernooij MW, Cordonnier C, Viswanathan A, Al-Shahi Salman R, Warach S, et al. Cerebral microbleeds: a guide to detection and interpretation. Lancet Neurol. (2009) 8:165-74. doi: 10.1016/S1474-4422(09)70 013-4

18. Scheltens P, Leys D, Barkhof F, Huglo D, Weinstein HC, Vermersch P, et al. Atrophy of medial temporal lobes on MRI in "probable" Alzheimer's disease and normal ageing: diagnostic value and neuropsychological correlates. J Neurol Neurosurg Psychiatry. (1992) 55:967-72. doi: 10.1136/jnnp.55. 10.967

19. Koedam EL, Lehmann M, van der Flier WM, Scheltens P, Pijnenburg YA, Fox N, et al. Visual assessment of posterior atrophy development of a MRI rating scale. Eur Radiol. (2011) 21:2618-25. doi: 10.1007/s00330-0112205-4

20. Ferreira D, Cavallin L, Granberg T, Lindberg O, Aguilar C, Mecocci $\mathrm{P}$, et al. Quantitative validation of a visual rating scale for frontal atrophy: associations with clinical status, APOE e4, CSF biomarkers and cognition. Eur Radiol. (2016) 26:2597-610. doi: 10.1007/s00330-015-4 101-9

21. Brown R, Benveniste H, Black SE, Charpak S, Dichgans M, Joutel A, et al. Understanding the role of the perivascular space in cerebral small vessel disease. Cardiovasc Res. (2018) 114:1462-73. doi: 10.1093/cvr/c vy113
22. Doubal FN, MacLullich AM, Ferguson KJ, Dennis MS, Wardlaw JM Enlarged perivascular spaces on MRI are a feature of cerebral small vessel disease. Stroke. (2010) 41:450-4. doi: 10.1161/STROKEAHA.109.56 4914

23. Riba-Llena I, Nafría C, Mundet X, López-Rueda A, Fernández-Cortiñas I, Jarca CI, et al. Assessment of enlarged perivascular spaces and their relation to target organ damage and mild cognitive impairment in patients with hypertension. Eur J Neurol. (2016) 23:1044-50. doi: 10.1111/ene. 12979

24. Hansen TP, Cain J, Thomas O, Jackson A. Dilated perivascular spaces in the Basal Ganglia are a biomarker of smallvessel disease in a very elderly population with dementia. AJNR Am J Neuroradiol. (2015) 36:893-8. doi: 10.3174/ajnr. A4237

25. Martinez-Ramirez S, Pontes-Neto OM, Dumas AP, Auriel E, Halpin A, Quimby $M$, et al. Topography of dilated perivascular spaces in subjects from a memory clinic cohort. Neurology. (2013) 80:1551-6. doi: 10.1212/WNL.0b013e31828 f1876

26. Paradise M, Crawford JD, Lam BCP, Wen W, Kochan NA, Makkar S, et al Association of dilated perivascular spaces with cognitive decline and incident dementia. Neurology. (2021) 96:e1501-11. doi: 10.1212/WNL.00000000000 11537

27. Puzo C, Labriola C, Sugarman MA, Tripodis Y, Martin B, Palmisano JN, et al. Independent effects of white matter hyperintensities on cognitive, neuropsychiatric, and functional decline: a longitudinal investigation using the National Alzheimer's Coordinating Center Uniform Data Set. Alzheimers Res Ther. (2019) 11:64. doi: 10.1186/s13195-019-0 521-0

28. Boyle PA, Yu L, Fleischman DA, Leurgans S, Yang J, Wilson RS, et al. White matter hyperintensities, incident mild cognitive impairment, and cognitive decline in old age. Ann Clin Transl Neurol. (2016) 3:791-800. doi: 10.1002/acn 3.343

29. Camarda C, Pipia C, Azzarello D, Battaglini I, Romeo G, Chiodi M, et al. Vascular risk factors, vascular diseases, and imaging findings in a hospital-based cohort of mild cognitive impairment types. Curr Alzheimer Res. (2018) 15:679-90. doi: 10.2174/156720501566618011911 0712

30. Bangen KJ, Thomas KR, Weigand AJ, Sanchez DL, DelanoWood L, Edmonds EC, et al. Pattern of regional white matter hyperintensity volume in mild cognitive impairment subtypes and associations with decline in daily functioning. Neurobiol Aging. (2020) 86:134-42. doi: 10.1016/j.neurobiolaging.2019. 10.016

31. van den Berg E, Geerlings MI, Biessels GJ, Nederkoorn PJ, Kloppenborg RP. White matter hyperintensities and cognition in mild cognitive impairment and Alzheimer's disease: a domain-specific metaanalysis. J Alzheimers Dis. (2018) 63:515-27. doi: 10.3233/JAD-17 0573

32. Yamanaka T, Uchida $\mathrm{Y}$, Sakurai $\mathrm{K}$, Kato $\mathrm{D}$, Mizuno $\mathrm{M}$, Sato $\mathrm{T}$, et al. Anatomical links between white matter hyperintensity and medial temporal atrophy reveal impairment of executive functions. Aging Dis. (2019) 10:711-8. doi: 10.14336/AD.2018. 0929

33. Wong FCC, Yatawara C, Low A, Foo H, Wong BYX, Lim L, et al. Cerebral small vessel disease influences hippocampal subfield atrophy in mild cognitive impairment. Transl Stroke Res. (2021) 12:284-92. doi: 10.1007/s12975-020-00 847-4

34. Basselerie H, Bracoud L, Zeestraten E, Bouguen E, Kiyasova V, Pueyo M, et al. Incident cerebral microbleeds detected by susceptibility weight-imaging help to identify patients with mild cognitive impairment progressing to Alzheimer's disease. J Alzheimers Dis. (2017) 60:253-62. doi: 10.3233/JAD170470

35. Valenti R, Del Bene A, Poggesi A, Ginestroni A, Salvadori E, Pracucci $\mathrm{G}$, et al. Cerebral microbleeds in patients with mild cognitive impairment and small vessel disease: the vascular mild cognitive impairment (VMCI)Tuscany study. J Neurol Sci. (2016) 368:195-202. doi: 10.1016/j.jns.2016. 07.018 
36. Zhang J, Liu L, Sun H, Li M, Li Y, Zhao J, et al. Cerebral microbleeds are associated with mild cognitive impairment in patients with hypertension. J Am Heart Assoc. (2018) 7:e008453. doi: 10.1161/JAHA.117.0 08453

37. Salvadori E, Poggesi A, Valenti R, Pracucci G, Pescini F, Pasi M, et al. Operationalizing mild cognitive impairment criteria in small vessel disease: the VMCI-Tuscany study. Alzheimers Dement. (2016) 12:40718. doi: 10.1016/j.jalz.2015.02.010

38. Caillaud M, Hudon C, Boller B, Brambati S, Duchesne S, Lorrain D, et al. Evidence of a relation between hippocampal volume, white matter hyperintensities, and cognition in subjective cognitive decline and mild cognitive impairment. J Gerontol B Psychol Sci Soc Sci. (2020) 75:1382-92. doi: 10.1093/geronb/ gbz120
Conflict of Interest: The authors declare that the research was conducted in the absence of any commercial or financial relationships that could be construed as a potential conflict of interest.

The handling editor declared a shared affiliation with several of the authors $\mathrm{XL}$ and $\mathrm{ZH}$ at time of review.

Copyright (C) $2021 \mathrm{Li}$, Shen, Jin, Jia, Zhou, Han, Zhang, Tong and Jiao. This is an open-access article distributed under the terms of the Creative Commons Attribution License (CC BY). The use, distribution or reproduction in other forums is permitted, provided the original author(s) and the copyright owner(s) are credited and that the original publication in this journal is cited, in accordance with accepted academic practice. No use, distribution or reproduction is permitted which does not comply with these terms. 